\title{
A note on investigating cooccurrence patterns and dynamics for many species, with imperfect detection and a log-linear modelling parameterisation
}

\author{
Darryl MacKenzie ${ }^{1}$, Jason Lombardi², and Michael Tewes ${ }^{2}$ \\ ${ }^{1}$ Proteus Wildlife Research Consultants \\ ${ }^{2}$ Texas A\&M University System
}

October 23, 2020

\begin{abstract}
1. Patterns in, and the underlying dynamics of, species cooccurrence is of interest in many ecological applications. Unaccounted for, imperfect detection of the species can lead to misleading inferences about the nature and magnitude of any interaction. A range of different parameterisations have been published that could be used with the same fundamental modelling framework that accounts for imperfect detection, although each parameterisation has different advantages and disadvantages. 2 . We propose a parameterisation based on log-linear modelling that does not require a species hierarchy to be defined (in terms of dominance), and enables a numerically robust approach for estimating covariate effects. 3. Conceptually the parameterisation is equivalent to using the presence of species in the current, or a previous, time period as predictor variables for the current occurrence of other species. This leads to natural, 'symmetric', interpretations of parameter estimates. 4. The parameterisation can be applied to many species, in either a maximum-likelihood or Bayesian estimation framework. We illustrate the method using camera trapping data collected on three mesocarnivore species in South Texas.
\end{abstract}

\section{Hosted file}

MacKenzie_etal_EcolEvo_v2.pdf available at https://authorea.com/users/369592/articles/488422a-note-on-investigating-cooccurrence-patterns-and-dynamics-for-many-species-withimperfect-detection-and-a-log-linear-modelling-parameterisation 